\title{
Modeling Contacts and Mobility for Wireless Mobile Networks
}

\author{
Ye $\operatorname{Tian}^{1}$ and Jiang $\mathrm{Li}^{2}$ \\ 1 Anhui Province Key Laboratory of High Performance Computing and Application, \\ School of Computer Science and Technology, \\ University of Science and Technology of China, Hefei, Anhui 230026, China, \\ yetian@ustc.edu.cn \\ 2 Department of Systems and Computer Science, \\ Howard University, Washington DC 20059, USA, \\ jli@scs.howard.edu
}

\begin{abstract}
The inter-contact time between mobile human-carried devices is one of the key metrics in studying the mobility-assisted routing paradigms for wireless mobile networks. Recent studies on this topic are focused on the aggregated distribution integrating all the device pairs' inter-contact times. In this work, we study real-world inter-contact times from a new aspect. By dividing the device pairs in groups and by investigating the group-wise inter-contact time distribution, we find that for the frequently contacting pairs, there are three segments on the distribution curve. We use superposition of three stochastic contact processes caused by the devices' independent movements and human intentions to explain the segments. Furthermore, we propose a mobility model, where each node uses a priority queue to schedule its movement, to emulate real-world human mobility. Theoretical analysis shows that the priority queue results in a power-law inter-contact time and we also demonstrate that our model seamlessly integrates the three contact processes. Finally, simulation study testifies that our mobility model could reproduce the contacts with their inter-contact times resembling the empirical ones, therefore is accurate in characterizing the complexity of the device contacts in wireless mobile networks.
\end{abstract}

Keywords: Inter-contact times, mobility model, wireless mobile networks

\section{Introduction}

In recent years, an infrastructure-free wireless mobile network composed of humancarried mobile devices such as cell phones and PDAs has been proposed (e.g. [1][2][3][4]). In such a network, as the topology is not always connected, a mobilityassisted routing paradigm (e.g. [2][3]) is applied, where a node receiving the data which is not destinated to itself holds the data, until it encounters the destination node or another node considered as the next hop for data delivery. Clearly 
in such a routing scheme, contacts between the devices are the only opportunity for communication, so the interval between consecutive contacts of a device pair, which is referred to as the inter-contact time, is essential.

In this paper, we investigate the inter-contact times obtained from a number of real-world datasets. Unlike previous studies, we group the device pairs according to their contacting frequencies and analyze the group-wise inter-contact time distribution. We find that for a frequently contacting group, there are three segments on its distribution curve, which is different from the previous observations [5][6]. Based on well-known results of human dynamics, we use superposition of three stochastic contact processes caused by devices' random movements and intentions of the humans carrying the devices to explain the observation.

We further present a mobility model for human-carried devices in wireless mobile network. In our model, each node uses a priority queue to schedule its movement. Theoretical analysis shows that power-law inter-contact times are resulted, and the model has integrated the three contact processes seamlessly. From simulation we find that the model generates contacts with their intercontact times of the same characteristics as the ones empirically observed. As the model exhibits the same characteristics as the ones observed from real world, it is very suitable to be applied in emulating and evaluating realistic wireless mobile networks composed of human-carried devices.

The remainder part of this paper is organized as the follows: Section 2 surveys the related work; We analyze the group-wise inter-contact time distribution in Section 3; In Section 4, the three stochastic contact processes are proposed, and we explain the empirical inter-contact time distribution with superposition of the three processes; We present the mobility model in Section 5, and analyze and examine the inter-contact times produced by our model in this section; Finally, Section 6 concludes this paper.

\section{Related Work}

In recent years, contact patterns of human-carried devices, in particular, the inter-contact times, are widely studied. By investigating a number of real-world datasets, Chaintreau et. al. find that the inter-contact time distributions aggregating all the device pairs are power-law, with the exponents smaller than one [5]. Recently, Karagiannis et. al. observe that a dichotomy exists on the complementary cumulative distribution function (CCDF) curves of the device pairs' aggregated inter-contact times [6]. Yoneki et. al. find that the inter-meeting times among meeting groups do not follow a power-law distribution[7]. A number of mobility models are proposed to interpret these observations. For example, Karagiannis et. al prove that for a random walk (RW) on a circuit, the distribution of two nodes' inter-meeting time scales as $n^{-1 / 2}$, when the inter-meeting time $n$ is sufficiently large, and there is an exponential tail[6]. Cai et. al. derive a similar result using more general random mobility models of random waypoint model (RWP) or RW on a two-dimensional area with finite boundary, and point out that the finite boundary is responsible for the exponential tail [8]. While some 
other general-purpose mobility models, such as the time-variant community mobility[9] and the levy walk model[10], also stick to the observations from the aggregated inter-contact times in [5] and [6]. On the other hand, although Conan et. al. address the heterogeneity in inter-contact times by studying contacts from single pairs of devices[11], however, the raw nature of pairwise contact data prohibits people to find more insights regarding device's contact pattern.

Meanwhile, it is every interesting to note that besides the inter-contact time, many recent studies[12][13][14][15] reveal that in human activities, the interevent time is power-law. Barabasi et. al. studies the time intervals between sending emails by a group of users, and reports that the distribution of the inter-email times by one user is power-law, of which the cumulative distribution function (CDF) could be modeled as $F(\tau) \sim \tau^{-2}$ [12]. In [13], [14], and [15], the intervals between human activities of visiting web sites, responding surface mails, and initializing finance transactions are examined, and it is observed that in these activities, the inter-event time distributions are all power-law, with a $\mathrm{CDF}$ as $F(\tau) \sim \tau^{-\alpha}$, where $\alpha \geq 2$.

\section{$3 \quad$ Studying Group-wise Inter-Contact Time}

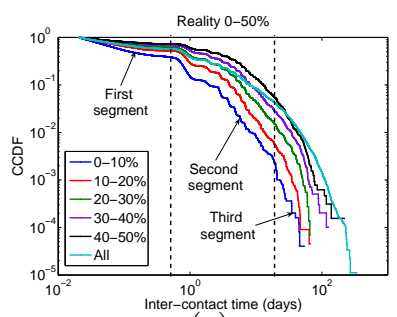

(a)

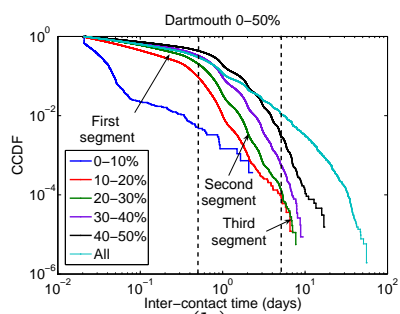

(b)

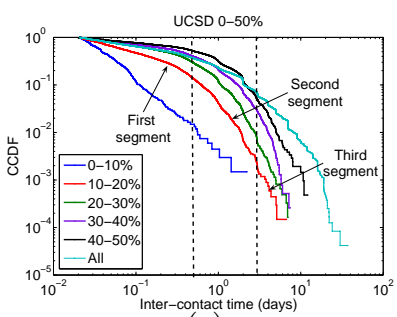

(c)

Fig. 1. Inter-contact time distribution of percentile groups in $0-50 \%$ for (a) Reality, (b) Dartmouth, (c) UCSD

We obtain and study the inter-contact times for pairs of devices in three realworld datasets, denoted as Reality[16], Dartmouth[17], and UCSD[18]. Unlike previous studies, in this work, we sort all the device pairs of a dataset in an ascending order based on their mean inter-contact times, and group the pairs in each ten percentiles. For example, by denoting the percentile group of "10-20", we mean the pairs with their mean inter-contact times between the first ten and the first twenty percents in this order.

We plot the aggregated inter-contact time distribution in CCDF for each percentile groups in $0-50 \%$ of the three datasets, as pairs in these groups contribute majority of the contacts. We also plot the distributions aggregating all the inter-contact times in the datasets for comparison. From the figures one can 
see that the distributions cannot be simply considered as exponential or powerlaw, suggesting the complexity in devices' contacting behaviors. Moreover, one can see that except for the "0-10" group in Dartmouth, all the other distribution curves of a same dataset closely resemble each other, suggesting that the device pairs in these groups were following similar patterns in making contacts.

To further investigate the frequently contacting device pairs, we focus on the percentile group with most contacts in each dataset, that is, the "0-10" group in Reality, the "20-30" group in Dartmouth, and the "10-20" group in UCSD. By carefully examining the figures, one can see that the distribution curve could be divided into three segments, as labeled in Fig. 1(a-c). The first segment's intercontact time is from 0 to a certain characteristic time, and the segment is almost a straight line with a slope rate less than one. The second segment's range is from the first characteristic time to another characteristic time, and the segment is also power-law, but its slope rate is no less than two, as demonstrated in the figure. The third segment is the rest of the distribution curve with inter-contact times larger than the second characteristic time. If the distributions were plotted under linear-log scale, one can see that the segments are straight lines, making them as exponential tails.

For less frequently contacting groups in $50-100 \%$, we also study their inter-contact times, and find that their inter-contact times are exponentially distributed. We do not show the results here for space reason.

\section{Interpreting the Inter-Contact Time Distribution}

\subsection{Source of the contacts}

To interpret the inter-contact time distributions observed from real-world datasets, we first discuss the causes of the device contacts. Generally, the contacts between human-carried devices are the result of human movement. Simplified random mobility models such as random waypoint model (RWP) or random walk (RW) are usually used to model human movement. Studies[19][20] prove that under these mobility models, the inter-meeting times are exponentially distributed. And people also use these simple random mobility models to explain power-law inter-contact times, as in [6] and [8]. However, we believe that these simple random mobility models are not sufficient to explain our observation in Section 3, because of the following reasons. First of all, [6] and [8] suggest that the powerlaw inter-contacts appear only when the inter-contact time is sufficiently large (Theorem 2 in [6] and Theorem 4 in [8]). However, from Fig. 1, one can see that the first power-law curve segment appears when the inter-contact time is less than half a day, which apparently is not very large. Moreover, [6] and [8] cannot explain the second power-law segment, which has a slope rate no less than two.

Meanwhile, recent studies show that in many human activities, the interevent time is power-law, of which the distribution function can be modeled as $F(\tau) \sim \tau^{-\alpha}$, with $\alpha \geq 2$. These activities include sending emails [12], visiting websites [13], responding surface mails [14], and initializing finance transactions 
[15]. Clearly, these findings strongly suggest that power-law inter-event time distribution with a slope rate larger than two is an universal principle in many human activities including human contacts. However, we must note that human contacting is a little different from other human activities such as sending emails and visiting websites, as in these activities, events are purely intentional results, but the contacts between two human beings may be unintentional (although each one moves intentionally).

Motivated by the above discussion, we use two factors to interpret the contacts between two human-carried devices: 1) independent random movement of devices that result in exponential inter-contact times; and 2) the same principle causing power-law inter-event times in other human activities such as email sending and website visiting. We deem the contacts caused by the first factor unintentional, as they are a byproduct of the device's independent random movement. The contacts caused by the second factor are regarded as intentional. Unintentional contacts may be the result of random movement of devices within a large (or "global") area as well as of movement restricted within a small (or "local") area. Contacts due to the former type of movement are likely to have larger inter-contact times than those due to the latter. In addition, "local" movement usually leads to "bursty" contacts, while "global" movement is more likely to incur "scattered" contacts. For example, the entire campus of a university can be viewed as a global area, while a building or a lab of the university can be viewed as a local area. Any two students on the campus have chances to encounter each other, but the contacts are scattered as the two students are unlikely to meet often in such a large area. On the other hand, when the two students are in a same building of the campus, they are more likely to make frequent contacts, until any of them leaves the building. The contacts are bursty as the time that the two students stay in the building is limited, compared with the time they stay on the campus. They will have another sequence of bursty contacts when they are within a local area simultaneously again.

Formally, we define three stochastic contact processes in the following.

- The first contact process is caused by device's local independent random movement, and the contacts are bursty. When there are contacts, the intercontact time distribution could be expressed as

$$
F_{1}(t)=1-e^{-\lambda_{l} t}
$$

with $\lambda_{l}>0$; as the contacts are in bursts, there exist long intervals without contacts between contact bursts. We refer to this process as local contact process (denoted as $L C P$ for short).

- The second contact process is intentional. Similar to the inter-event time in other human activities, the inter-contact time is power-law. To make our analysis mathematically tractable, we use the shifted Pareto distribution to express the inter-contact time distribution as

$$
F_{2}(t)=1-\left(1+\frac{t}{t_{m}}\right)^{-\alpha}, \alpha \geq 2
$$


where $t_{m}>0$ and $\alpha \geq 2$, and $t_{m}$ is the smallest value $t$ can take. As it is human's intention that causes the contacts, the process is refer to as the intentional contact process (denoted as ICP for short).

- The third contact process is caused by device's global independent random movement, and the inter-contact time distribution could be expressed as

$$
F_{3}(t)=1-e^{-\lambda_{g} t}
$$

with $\lambda_{g}>0$. We refer to this process as the global contact process (denoted as $G C P$ for short).

Given the three contact processes, we assume that LCP's mean inter-contact time, $1 / \lambda_{l}$, is much smaller than that of the other two processes; while GCP's mean inter-contact time, $1 / \lambda_{g}$, is much larger than that of the other two. For $\mathrm{ICP}$, as its inter-contact time distribution is power-law, it has many very short inter-contact times as well as many inter-contact times that are very long. The underlying intuition of our assumption is that if two persons are in a small area simultaneously, they are more likely to encounter each other. In a large global area, unintentional contacts happen less frequently than intentional ones (especially those between people with certain relationship).

In the following subsections, we show that the three segments on the distribution curves observed in Section 3 could be explained by the superposition of the three stochastic contact processes aforementioned. By superposition, we mean that events from different source processes are undifferentiated. Strict definition can be found in [21].

\subsection{Explaining the knob between the second and the third segments}

We first explain the knob between the second the third segments on the intercontact time distributions in Fig. 1. As the second characteristic time separating the two segments is from days to tens of days, which is sufficiently large, we ignore the first contact process which produces small inter-contact times in bursts, and focus on the second and the third contact processes. Assuming that the two processes are independent and renewal, with their inter-contact times following the distributions of $F_{2}(t)$ and $F_{3}(t)$ respectively, the inter-contact time distribution of the superposition process from ICP and GCP could be expressed as [21]

$$
R_{s}(t)=\frac{\bar{v}_{2} \bar{v}_{3}}{\bar{v}_{2}+\bar{v}_{3}}\left(\psi_{2}(t) R_{3}(t)+\psi_{3}(t) R_{2}(t)\right)
$$

where $R_{i}(t)=1-F_{i}(t), \bar{v}_{i}$ is the mean contact rate, and $\psi_{i}(t)=\int_{t}^{\infty} R_{i}(u) d u$, for $i=2,3$.

By applying Equ. (1), we can obtain expression of the inter-contact time distribution for the superposition process. Taking logarithm, we have

$$
\log R_{s}(t)=\log \left(1+\frac{\lambda_{g} t}{\lambda_{g} t_{m}+\alpha-1}\right)-\lambda_{g} t_{m} \frac{t}{t_{m}}-\alpha \log \left(1+\frac{t}{t_{m}}\right)
$$


Since the mean inter-contact time of GCP is much larger than ICP, we have $(\alpha-1) \gg \lambda_{g} t_{m}$. We consider two cases.

- Case I, the inter-contact time is moderately larger than $t_{m}$, but is much smaller than $\frac{\alpha-1}{\lambda_{g}}$, i.e., $t_{m}<t \ll \frac{\alpha-1}{\lambda_{g}}$. First of all we know that $\frac{t}{t_{m}}>$ $\log \left(1+\frac{t}{t_{m}}\right)$. On the other hand, as $\alpha>(\alpha-1)$ and $(\alpha-1) \gg \lambda_{g} t_{m}$, we have $\alpha \gg \lambda_{g} t_{m}$. Mutipling $\alpha$ with $\log \left(1+\frac{t}{t_{m}}\right)$ and $\lambda_{g} t_{m}$ with $\frac{t}{t_{m}}$, recall that $\frac{t}{t_{m}}$ is only moderately larger than $\log \left(1+\frac{t}{t_{m}}\right)$ but $\alpha \gg \lambda_{g} t_{m}$, it should have $\alpha \log \left(1+\frac{t}{t_{m}}\right) \gg \lambda_{g} t_{m} \frac{t}{t_{m}}$. In addition, as $\lambda_{g} t \ll \alpha-1, \frac{\lambda_{g} t}{\lambda_{g} t_{m}+\alpha-1} \rightarrow 0$, so $\log \left(1+\frac{\lambda_{g} t}{\lambda_{g} t_{m}+\alpha-1}\right) \approx \frac{\lambda_{g} t}{\lambda_{g} t_{m}+\alpha-1} \ll \lambda_{g} t_{m} \frac{t}{t_{m}} \ll \alpha \log \left(1+\frac{t}{t_{m}}\right)$. Since $\log \left(1+\frac{\lambda_{g} t}{\lambda_{g} t_{m}+\alpha-1}\right)$ and $\lambda_{g} t_{m} \frac{t}{t_{m}}$ are much smaller than $\alpha \log \left(1+\frac{t}{t_{m}}\right)$, by ignoring them we can see that for this case $\log R_{s}(t) \approx-\alpha \log \left(1+\frac{t}{t_{m}}\right) \approx$ $-\alpha \log \left(\frac{t}{t_{m}}\right)$. The distribution is power-law with a slope rate of $\alpha$.

- Case II, the inter-contact time is much larger than $\frac{\alpha}{\lambda_{g}} \log \left(1+\frac{t}{t_{m}}\right)$, i.e., $t \gg$ $\frac{\alpha}{\lambda_{g}} \log \left(1+\frac{t}{t_{m}}\right)$. Obviously, for this case $\lambda_{g} t_{m} \frac{t}{t_{m}} \gg \alpha \log \left(1+\frac{t}{t_{m}}\right)$, and since $\alpha \geq 2$, we have $\frac{t}{t_{m}}>\frac{\lambda_{g} t}{\lambda_{g} t_{m}+\alpha-1}$, so it is easy to see that $\alpha \log \left(1+\frac{t}{t_{m}}\right)>$ $\alpha \log \left(1+\frac{\lambda_{g} t}{\lambda_{g} t_{m}+\alpha-1}\right)>\log \left(1+\frac{\lambda_{g} t}{\lambda_{g} t_{m}+\alpha-1}\right)$. As $\lambda_{g} t$ is much larger than $\alpha \log \left(1+\frac{t}{t_{m}}\right)$ and $\log \left(1+\frac{\lambda_{g} t}{\lambda_{g} t_{m}+\alpha-1}\right)$, by ignoring them, we have $\log R_{s}(t) \approx$ $-\lambda_{g} t$, that is, the distribution has an exponential tail with a rate of $\lambda_{g}$.

\subsection{Explaining the knob between the first and the second segments}

We then explain the knob between the first and the second segments observed in Fig. 1. As we are interested in the inter-contact time of the superposition process around the first characteristic time where the knob appears, which is about half a day, we ignore the process of GCP for its large mean inter-contact time, and only consider the contact processes of LCP and ICP. Unfortunately, as LCP is not renewal, we cannot apply Equ. (1) directly.

Meanwhile, as the mean inter-contact time of LCP is much less than ICP, and there are long intervals without contacts between its contact bursts, we use a two-state Markov chain for LCP to produce bursts of contacts. In this model, LCP may be in ON or OFF state: when at the ON state, with probability $p$ a contact with a random interval to the next contact following the distribution of $F_{1}(t)$ is generated, and the model stays at the ON state; LCP changes to the OFF state with probability $1-p$. At the OFF state, there is no contacts for an interval of length $L$, and at the end of the interval, the process stays at the OFF state with a probability of $q$ without a contact, and goes to the ON state to have a new contact with a probability $1-q$. We further assume that the influence of the contacts from LCP on the inter-contact times of ICP, especially 
the long inter-contact times, is neglectable. Therefore, the inter-contact times of LCP and ICP can be considered separately. Specifically, the distribution of the inter-contact time in the superposition process can be approximated as

$$
F_{c}(t) \approx p_{1} \cdot F_{1}(t)+p_{2} \cdot F_{2}(t)= \begin{cases}1-p_{1} e^{-\lambda_{l} t}-p_{2}\left(\frac{t}{t_{m}}\right)^{-\alpha}, & t>t_{m} \\ 1-e^{-\lambda_{l} t}, & t \leq t_{m}\end{cases}
$$

for $\lambda_{l}, t_{m}>0$, and $\alpha \geq 2$

Let $R_{c}(t)=1-F_{c}(t)$. Since the mean inter-contact time of LCP is much less than ICP, we have $t_{m} \lambda_{l} \ll \frac{\alpha-1}{\alpha}$. Two cases are considered.

- Case I, the inter-contact time $t$ is much smaller than $t_{m}$, i.e., $t \ll t_{m}$. In this case, $\log R_{c}(t) \approx \log e^{-\lambda_{l} t}$, the distribution should be exponential. However, as here we do not consider the distribution tail, we show that $R_{c}(t)$ can also be approximated by a power-law distribution function, as shown in the following. Let $t_{m}^{\prime}$ be a constant smaller than $t_{m}$, and rewrite $e^{-\lambda_{l} t}$ as $e^{-\lambda_{l} \cdot t_{m}^{\prime} \cdot \frac{t}{t_{m}^{\prime}}}$, since $e^{-\lambda_{l} \cdot t_{m}^{\prime} \cdot \frac{t}{t_{m}^{\prime}}} \approx\left(1+\frac{t}{t_{m}^{\prime}}\right)^{-\lambda_{l} \cdot t_{m}^{\prime}}$ for $\frac{t}{t_{m}^{\prime}}$ sufficiently small (note that $\log (1+x) \approx x$ for $x \rightarrow 0), \log R_{c}(t) \approx-\lambda_{l} t_{m}^{\prime} \log \left(1+\frac{t}{t_{m}^{\prime}}\right) \approx$ $-\lambda_{l} t_{m}^{\prime} \log \left(\frac{t}{t_{m}^{\prime}}\right)$, which is power-law with a slope rate of $\lambda_{l} t_{m}^{\prime}$. As $t_{m} \lambda_{l} \ll$ $\frac{\alpha-1}{\alpha}<1$ and $t_{m}^{\prime}$ can take any value smaller than $t_{m}$, clearly $\lambda_{l} t_{m}^{\prime}<1$. This explains the first segment.

- Case II, the inter-contact time $t$ is larger than $t_{m}$, i.e., $t>t_{m}$. For this case, $R_{c}(t) \approx p_{1} \cdot e^{-\lambda_{l} t}+p_{2} \cdot\left(\frac{t}{t_{m}}\right)^{-\alpha}$. As an exponential distribution decays much faster than a power-law distribution function, when $t$ is sufficiently large, we can ignore $p_{1} e^{-\lambda_{l} t}$ and rewrite Equ. (3) as $\log R_{c}(t)=\log \left(p_{2} \cdot\left(\frac{t}{t_{m}}\right)^{-\alpha}\right)=$ $\log p_{2}-\alpha \log \left(\frac{t}{t_{m}}\right)$. The distribution is power-law with a slope rate of $\alpha$ $(\alpha \geq 2)$. This explains the second distribution curve segment.

From the above analysis, one can see than $t_{m}$ actually is the first characteristic time in Section 3 , thus $t_{m} \approx$ half a day. In addition, we believe that the first power-law curve segment observed in Fig. 1 should be the integration of a number of contact processes as LCP, as a device may be restricted in different local areas during different times. From [22], we know that synthesizing a power-law distribution with a number of exponential distributions is possible.

To validate our analysis, we simulate three contact processes of LCP, ICP and GCP with their inter-contact time distributions expressed as $F_{1}, F_{2}(t)$ and $F_{3}(t)$ using different parameter values of $\lambda_{l}, \alpha, t_{m}$, and $\lambda_{g}$, and plot the intercontact time distribution of the superposition process in Fig. 2. One can see from the figure that on the distribution curve there is a power-law segment with a slope rate smaller than one, followed by a power-law segment with a slope rate of approximately two, and the rest of the superposition process's distribution can be viewed as an exponential tail. Moreover, the value of $t_{m}$ determines the 


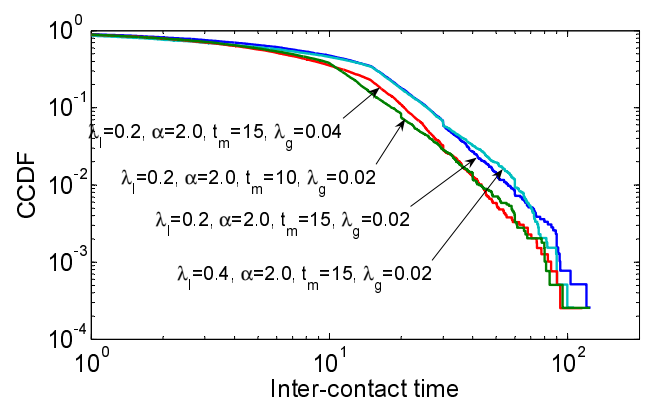

Fig. 2. Inter-contact time distribution of the superposition process of LCP, ICP, and GCP.

position of the first knob. In short, the inter-contact time distribution in Equ. (2) and Equ. (3) explain the two knobs on the inter-contact time distribution curves observed in Fig. 1.

\subsection{Verification by the real-world datasets}

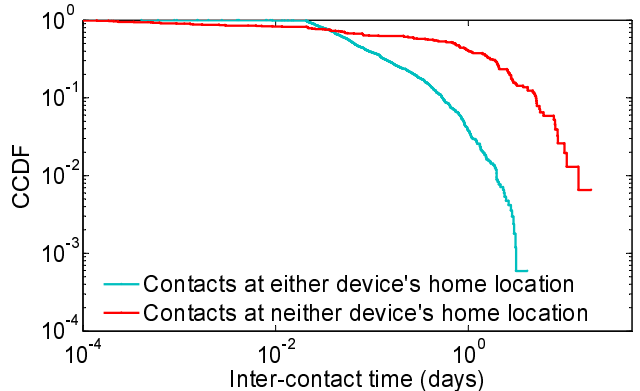

(a)

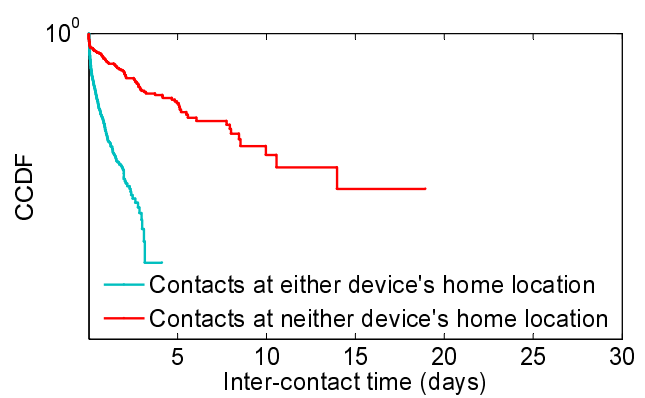

(b)

Fig. 3. Inter-contact time distribution of contacts at (a) either device's home location, and (b) neither device's home location

In the above discussions, we used the superposition of three contact processes to interpret the inter-contact time distributions observed in Section 3. In this section, we justify our explanation by analyzing the real-world datasets. Unfortunately, for all the currently available datasets, we cannot tell the source of a contact (e.g., intentional or unintentional), therefore we have to find other methods to infer the contacts in a real-world dataset.

Our approach is to consider the location where contacts took place. In UCSD, as contacts are inferred by simultaneous AP association, we recorded the location 
information (i.e. AP) for each contact. We found that every device stayed at one particular location for most of the time. This location is considered as its "home" location. For example, it is observed that on average a device in UCSD spent $90.9 \%$ of all its time at its home location. In our approach, we exploited home locations to filter out some of the unintentional contacts caused by global device movement. Specifically, given a pair of frequently contacting devices with different home locations, we treat the contacts at either home location and the contacts at other locations separately. We collect the inter-contact times for the two types of contacts for all of the device pairs in the "10-20" percentile group in UCSD, and plot their inter-contact time distributions in Fig. 3, using a log-log scale in Fig. 3(a) and a linear-log scale in Fig. 3(b). The figures show that for the contacts at either device's home location, the power-law segments are prominent, indicating that most of the contacts at either device's home are caused by device's local independent movement and human's intention, while the inter-contact time distribution of the contacts at non-home locations is exponential, suggesting that the contacts are caused by device's global independent movement.

\section{$5 \quad$ Mobility Model}

In this section, we present a mobility model based on our observation and interpretation of the human-carried device contacts discussed in previous sections. Under this model, the distribution of inter-contact time between nodes closely resembles the distributions observed in the real-world datasets discussed in Section 3 , with two power-law segments followed by an exponential tail. With these properties, our model can be applied for design and evaluation of mobility-assisted routing paradigms for wireless mobile networks.

\subsection{Model description}

In our mobility model, we assume that nodes are equipped with omni-antennas and the disk communication model applies: a node can communicate with another if their distance is less than a communication range of $r$. Moreover, each node is associated with a region called its home region. The size of the home region is much smaller than the entire network area, but should be larger than a node's communication area (i.e., a disk of size $\pi r^{2}$ ). In our implementation, the network area is a square of $L \times L$, and the home region is a $l \times l$ square where $L>l$. Each node knows the home region of itself as well as that of all the other nodes (referred to as foreign regions for this node) in the network. It stays in its home region or visits another node's home region according to a two-state Markov chain. Specifically, when a node is in a region, either home or foreign, the time is divided into intervals of length $T_{l}$. At the end of each interval, if the node is in its home region, it stays at the home region during the next interval with a probability of $p_{H}$, and visits a foreign region with a probability of $1-p_{H}$. Similarly, when a node is in a foreign region, it stays in the same foreign region with a probability of $p_{F}$, and returns to its home region with a 
probability of $1-p_{F}$. When in a region, a node performs random movement. In our implementation, we uses the random waypoint mobility model without pause.

The selection of foreign region for a node to visit is critical. In our model, each node keeps a queue of visiting plans. A visiting plan keeps the information of the node's ID of which the home region is to be visited, as well as a priority value uniformly chosen from $[0,1)$. When a node decides to visit a foreign region, it selects and removes the visiting plan in its queue with the highest priority, and moves to a randomly chosen position in the corresponding foreign region directly. When a node at a foreign region returns to its home region, it also moves to a randomly chosen position in its home region directly. At the same time, a new visiting plan with a randomly selected priority to the foreign region the node is currently leaving is inserted into its visiting plan queue. Clearly, the queue is a priority queue. In a strict priority queue, plans of low priorities may never be accessed (and removed). Therefore, we introduced randomness as follows: when selecting a visiting plan to remove, the node chooses a plan of the highest priority with a probability $p_{r}$, and if the plan of the highest priority is not chosen, the node chooses the plan with the second highest priority with probability $p_{r}$, and so on, until a plan is selected.

Finally, when a node is moving, either staying in a region or moving towards another region, the speed is randomly selected from a range of $\left[v_{\min }, v_{\max }\right]$ with a mean value as $\bar{v}$.

\subsection{Analysis}

In this section, we prove that power-law inter-contact times exist for node pairs under our presented model, and show that our model seamlessly integrates contact processes of LCP, ICP, and GCP between node pairs.

We first consider the interval between the executions of two consecutive visiting plans by a node. The interval includes the time for the node to move to the foreign region of the first plan, the time spent by the node to stay in the foreign region, the time for the node to return to its home region, and the time it stays in the home region of itself, until it decides to select and remove the next visiting plan. Mathematically, the expectation of the interval $T$ could be expressed as

$$
E[T]=\frac{T_{l}}{\left(1-p_{F}\right)^{2}}+2 \frac{\bar{D}}{\bar{v}}+\frac{T_{l}}{\left(1-p_{H}\right)^{2}}
$$

where $\frac{T_{l}}{\left(1-p_{H}\right)^{2}}$ and $\frac{T_{l}}{\left(1-p_{F}\right)^{2}}$ are the expected times for a node to stay in home and foreign regions respectively, and $\bar{D}$ is the mean distance between the node's home region and a foreign region.

Given a node, we denote the time $\tau$ a visiting plan spends in queue, i.e., the period between the moment it is inserted and the moment it is removed. We assume that the visiting plan queue is a strict priority queue for simplifying our analysis. Suppose that there are $M$ plans in the queue. Over a period sufficiently long, the probability that a plan of priority $\rho$ gets selected is $\rho^{M}$, which is the 
probability that $\rho$ is the largest value among $M$ uniformly distributed random variables in $[0,1)$. Assuming that the distributions of the priorities of the plans in the queue at the movements of plan selection are independent, then for a plan with priority $\rho$, the probability that it gets selected at the first plan selecting time after its insertion should be $\rho^{M}$; and if it is not selected, it must wait for a time of $T$ and gets selected with the same probability $\rho^{M}$, and so on, until it is selected and removed. Therefore we could express the mean waiting time of a visiting plan with priority $\rho$ as

$$
\tau(\rho)=\sum_{i=0}^{\infty}(i+1) \times E[T] \times \rho^{M} \times\left(1-\rho^{M}\right)^{i}=\frac{E[T]}{\rho^{M}}
$$

Consider all the plans with their priorities in a small range of $[\rho, \rho+d \rho)$. The probability that they are inserted into the queue can be expressed as $f(\rho) d \rho$, where $f(\rho)$ is the probability density function of the priority distribution, which is uniform in our model; and for all the plans with their waiting times in a small range of $[\tau(\rho), \tau(\rho)+d \tau)$, the rate of their removals is $P(\tau) d \tau$, where $P(\tau)$ is the probability density function of the waiting time. Applying Little's law, it is easy to see that

$$
\frac{f(\rho) d \rho}{E[T]}=P(\tau) d \tau \Rightarrow P(\tau)=\frac{f(\rho)}{E[T]} \frac{d \rho}{d \tau}
$$

From Equ. (4) we know $\frac{d \rho}{d \tau}=-\frac{1}{n} \frac{E[T]^{1 / M}}{\tau^{1+1 / M}}$, and since priority is uniformly distributed, $f(\rho)$ is a constant. Therefore $P(\tau) \sim \frac{1}{\tau^{1+1 / M}}$, and the probability density function is power-law with an exponent of $1+\frac{1}{M}$. As priorities are randomly selected each time when a visiting plan is inserted, under our mobility model, the distribution of inter-visiting time to a specific foreign region by a node is power-law, of which the slope rate under log-log scale is $2+\frac{1}{M}$, which is larger than two.

Clearly, time between two visits of a node to another node's home region is not strictly the inter-contact time. Nonetheless, if we properly configure the model parameters, inter-visit time should be a good approximation of intercontact time between two nodes. In other words, our mobility model contains the contact process of ICP, i.e., the contact process with power-law inter-contact times in Section 4. Moreover, as the minimum interval between two consecutive visits to a foreign region by a node is $T, T$ actually is the characteristic time of the first knob. In other words, for the ICP's inter-contact time distribution function $F_{2}(t), t_{m}=E[T]$ and $\alpha=2+\frac{1}{M}$.

We also show that our mobility model integrates the contact processes of LCP and GCP as well. Recall that when two nodes are in the same region, as they move independently and the region size is small, they will make frequent contacts. That corresponds to LCP where contacts are frequent and inter-contact times are exponential distributed. More specifically, at any time the probability that two devices in a same region meet with a probability of $P_{l}=\frac{2 r \delta \bar{v}}{l^{2}}$, where $2 r \delta \bar{v}$ is the area covered by a device in unit time when moving with the relative average speed of the device to the other device $\delta \bar{v}$. From [20], it is known that 
$\delta \approx 1.27$. It is well-known that when the unit time is small, the geometric distribution could be approximated as an exponential distribution, therefore for the inter-contact time distribution of the LCP contact process $F_{1}(t), \lambda_{l}=\frac{2 r \delta \bar{v}}{l^{2}}$.

The GCP process is also taken care of. As nodes are moving back and forth between its home region and a number of foreign regions, which are randomly located over the entire network area, when there are many foreign regions, traveling between them can be viewed as a global random movement. Therefore the proposed mobility model also contains the contact process of GCP with long inter-contact times exponentially distributed. Similarly, for the inter-contact time distribution of the GCP contact process $F_{3}(t)$, we have $\lambda_{g}=\frac{2 r \delta \bar{v}}{L^{2}}$.

\subsection{Validation}

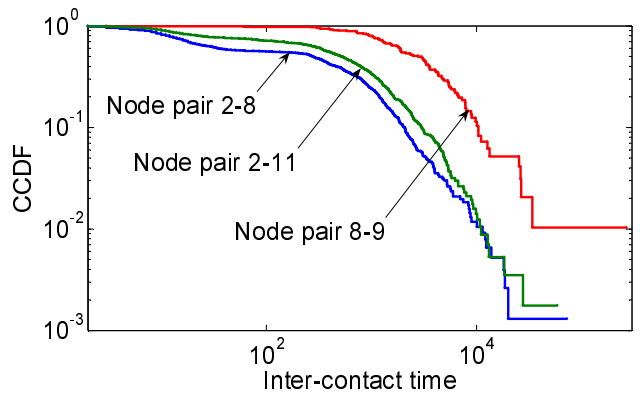

(a)

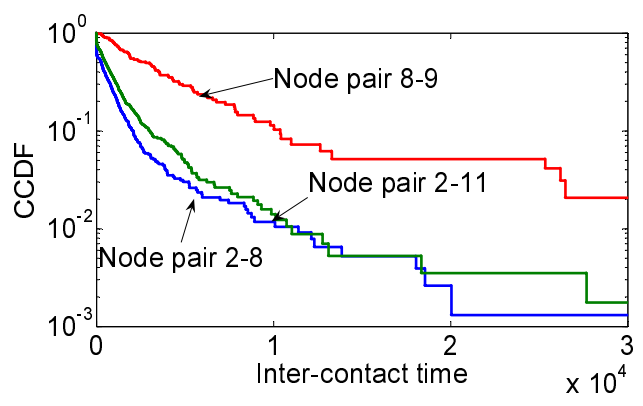

(b)

Fig. 4. Inter-contact time distributions of node pairs in mobility model

In this section, we show that our mobility model captures the complexity of the contact behaviors, especially the inter-contact times, of human-carried devices. We implement a simulator of wireless mobile networks, in which nodes move according to the proposed mobility model. In our simulation, we create a network containing 20 nodes that move within a global area of size $800 \times 800$, the home/foreign reign size is $50 \times 50$, the communication range is $r=10$, the length of the interval is $T_{l}=20$, the minimum and the maximum speeds are $v_{\text {min }}=3$ and $v_{\max }=5$, and transition probabilities of the two-state Markov chain are $p_{H}=0.8$ and $p_{F}=0.6$, and the probability $p_{r}$ is set as 0.8 . Furthermore, in the simulation we divide the 20 nodes into two groups, each of 10 nodes. A node in our simulation only visits the home regions of the nodes in the same group. In this way we have two relationships between nodes: nodes in a same group and nodes in different groups.

We investigate the inter-contact time of different node pairs. In particular, three representative node pairs are studied: the pair of node 2 and node 8 , the pair of node 8 and node 9 , and the pair of node 2 and node 11 . The nodes in the 
pair of pair 2-8, and 8-9, are respectively in the same group, and they visit each other's home region according to their priority queues to make contacts. While the nodes in pair 2-11 are in different groups, so they do not make such contacts. Fig. 4 plots the inter-contact time distributions of the three node pairs. From the figure one can see that for the pairs in the same group, the curves of the inter-contact time distributions closely resemble the distributions in Fig. 1 , as there are two power-law segments followed by an exponential tail on the distribution curve. The inter-contact times of the node pair 2-11 are exponentially distributed. This observation showed that our model can reproduce the inter-contact time distributions very similar to the distributions of frequently and infrequently contacting device pairs observed in Section 3.

\section{Conclusion}

In this paper, we focused on examining and interpreting the inter-contact times between mobile human-carried devices. By investigating a number of real-world datasets, we found that the inter-contact time distributions of frequently-contacting percentile groups have three segments on their distribution curves. Based on existing knowledge of random movements and human activities, we conjectured that there are two reasons causing device contacts: the device's independent movement and the human's intention. We used superposition of three stochastic contact processes, i.e., the temporal local contact process, the intentional contact process, and the global contact process to explain the inter-contact time distributions observed. Based on our analysis, we proposed a mobility model for human-carried devices, and showed that the mobility model seamlessly integrates the three contact processes. Especially, we theoretically proved that the priority queue in our model generates power-law inter-visiting times. Finally, simulation experiment showed that our mobility model reproduces the contacts between node pairs with their inter-contact time distributions highly similar to the ones observed in real-world datasets, suggesting that the model could be applied in designing and evaluating realistic wireless mobile networks composed of human-carried devices.

\section{Acknowledgement}

This work was funded by the Provincial Foundation for Excellent Young Talents of Colleges and Universities of Anhui Province (No. 2010SQRL006ZD) and the Program for Innovative Research Team at University of Science and Technology of China, and was funded in part by US NSF grant CNS-0832000.

\section{References}

1. Chaintreau, A., Hui, P., Crowcroft, J., Diot, C., Gass, R., Scott, J.: Pocket switched networks: real-world mobility and its consequences for opportunistic forwarding. Technical Report UCAM-CL-TR-617, University of Cambridge (2005) 
2. Hui, P., Crowcroft, J., Yoneki, E.: Bubble Rap: social-based forwarding in delay tolerant networks. In: Proc. of ACM MobiHoc'08, Hong Kong, China (May 2008)

3. Grossglauser, M., Tse, D.N.C.: Mobility increases the capacity of ad hoc wireless networks. IEEE/ACM Trans. Networking 10(4) (2002) 477-486

4. Jain, S., Fall, K., Patra, R.: Routing in a delay tolerant network. In: Proc. of ACM SIGCOMM'04, Portland, OR, USA (August 2004)

5. Chaintreau, A., Hui, P., Diot, C., Gass, R., Scott, J.: Impact of human mobility on the design of opportunistic forwarding algorithms. In: Proc. of IEEE INFOCOM'06, Barcelona, Spain (April 2006)

6. Karagiannis, T., Boudec, J.Y.L., Vojnovic, M.: Power law and exponential decay of inter contact times between mobile devices. In: Proc. of ACM MobiCom'07, Montreal, Canada (September 2007)

7. Yoneki, E., Greenfield, D., Crowcroft, J.: Dynamics of inter-meeting time in human contact networks. In: Proc. of the International Conference on Advances in Social Networks Analysis and Mining, Athens, Greece (July 2009)

8. Cai, H., Eun, D.Y.: Crossing over the bounded domain: from exponential to powerlaw inter-meeting time in manet. In: Proc. of ACM MobiCom'07, Montreal, Canada (September 2007)

9. Hsu, W., Spyropoulos, A., Psounis, K., Helmy, A.: Modeling time-variant user mobility in wireless mobile networks. In: Proc. of IEEE INFOCOM'07, Anchorage, AK, USA (May 2007)

10. Rhee, I., Shin, M., Hong, S., Lee, K., Chong, S.: On the levy-walk nature of human mobility. In: Proc. of IEEE INFOCOM'08, Phoenix, AZ, USA (April 2008)

11. Conan, V., Leguay, J., Friedman, T.: Characterizing pairwise inter-contact patterns in delay tolerant networks. In: Proc. of the 1st international conference on Autonomic computing and communication systems, Rome, Italy (October 2007)

12. Barabasi, A.L.: The origin of bursts and heavy tails in human dynamics. Nature 435 (2005) 207-211

13. Dezso, Z., Almaas, E., Lukacs, A., Racz, B., Szakadat, I., Barabasi, A.L.: Dynamics of information access on the web. Phys. Rev. E 73 (2006) 066132

14. Oliveira, J.G., Barabasi, A.L.: Human dynamics: the correspondence patterns of Darwin and Einstein. Nature 437 (2005) 1251

15. Plerou, V., Gopikrishnan, P., Amaral, L.A.N., Gabaix, X., Stanley, H.E.: Economic fluctuations and anomalous diffusion. Phys. Rev. E 62 (2000) 3023-3026

16. Reality: MIT Reality Mining Project http://reality.media.mit.edu.

17. Kotz, D., Henderson, T., Abyzov, I.: CRAWDAD trace set dartmouth/campus/movement (v. 2005-03-08). Downloaded from http://crawdad.cs.dartmouth.edu/dartmouth/campus/movement (March 2005)

18. UCSD: UCSD WTD project http://sysnet.ucsd.edu/wtd.

19. Groenevelt, R., Nain, P., Koole, G.: The message delay in mobile ad hoc networks. Perform. Eval. 62(1-4) (2005) 210-228

20. Spyropoulos, T., Psounis, K.: Performance analysis of mobilityassisted routing. In: Proc. of ACM MobiHoc'06, Florence, Italy (May 2006)

21. Torab, P., Kamen, E.W.: On approximate renewal models for the superposition of renewal processes. In: Proc. of IEEE ICC'01, Helsinki, Finland (June 2001)

22. Feldmann, A., Whitt, W.: Fitting mixtures of exponentials to long-tail distributions toanalyze network performance models. In: Proc. of IEEE INFOCOM'97. (April 1997) 